\title{
Erbium in a-Si:H
}

\author{
Leandro R. Tessler \\ Instituto de Física "Gleb Wataghin", UNICAMP, \\ C. P. 6165, 13083-970, Campinas, SP, Brazil \\ tessler@ifi.unicamp.br
}

Received 27 May, 1999

\begin{abstract}
A review of the current status of research on $\mathrm{Er}^{3+}$ doped hydrogenated amorphous silicon (a-Si:H) is presented. Er has been introduced in a-Si:H and a-SiO $x: H$ by ion implantation, co-sputtering and PECVD. In all cases, the characteristic atomic-like intra- $4 f{ }^{4} I_{13 / 2} \rightarrow{ }^{4} I_{15 / 2}$ photoluminescence emission at $\sim 1.54 \mu \mathrm{m}$ is observed at room temperature. The $\mathrm{Er}^{3+}$ luminescence probability is determined by the local neighborhood of the ions. Therefore, local probes like EXAFS and Mössbauer spectroscopy have yielded very important information. A discussion of excitation processes, electroluminescence, and electronic doping effects, is also presented.

PACS Number(s): 71.23.Cq, 73.61.Jc, 81.05.Gc
\end{abstract}

\section{Introduction}

The global telecommunications network has experienced an enormous expansion in the last few years. The present bandwidth requirements are beyond the capability of conventional electronic technology. One solution for this problem has been the development of photonics, the use of light for information processing and distribution. Silica-based fiber optics have allowed a bandwidth increase of orders of magnitude relative to microwaves or cabled communications. Commercial systems are already flirting with the terabit/sec barrier on a single fiber[1]. In the laboratory, 2.6 Tbit/sec has been achieved over $120 \mathrm{~km}$ of fiber[2]. Conventional silica optical fibers present the minimum attenuation losses at wavelengths near $1.5 \mu \mathrm{m}$, defining the so-called "third spectral window" (the other two are respectively at 0.9 and $1.3 \mu \mathrm{m}$ ) An important challenge to materials and semiconductors scientists nowadays is to develop light sources and detectors operating at these wavelengths at low cost and high reliability. Moreover, it would be advantageous if the new devices would be compatible with the well established silicon microelectronic technology. Currently the only reliable light sources for fiber optics are the III- $V$ nearband edge lasers, whose high cost render large scale consumer applications prohibitive. An important alternative consists on the use of rare-earth based light emitters, which operate based on the atomic-like tran- sitions within the incomplete $4 f$ levels of these elements. A great effort has been devoted to the study of rare-earth doped semiconductors[3, 4], specially erbium doped silicon that emits light in the third spectral window range. The recent developments are inducing the emergence of a new field of research, that of siliconbased optoelectronics[5].

The rare earth elements are distinguished by their incomplete internal $4 f$ shell. When diluted in solid hosts, the rare earth atoms become almost always trivalent ions, losing the two $6 s$ and one $4 f$ electrons. The $4 f$ electrons are shielded from external fields by the two remaining electronic shells with larger radial extension $\left(5 s^{2} 5 p^{6}\right)$. The intra $4 f$ electronic transitions are thus atomic-like, weakly dependent on the details of the local chemistry. However, $f-f$ transitions are electric dipole forbidden $(\Delta l=0)$ in free ions. Only when the rare earth ions are incorporated in a solid host which breaks inversion symmetry and mixes states of opposite parity is the transition allowed. The transition probability is, therefore, strongly dependent on the chemical neighborhood of the ions. Among the rare earths, $\mathrm{Er}^{3+}$ has been the most widely studied because the transition between its two lowest spin-orbit levels corresponds to the emission of a photons with wavelengths close to $1.54 \mu \mathrm{m}[6]$. The local field splits the spin-orbit manifolds into a number of Stark levels. The degeneracy $g$ of each state is given by $g=J+1 / 2$, so there are 7 initial and 8 final possible states, which may be 
broadened both homogeneously or inhomogeneously[7]. A schematic representation of the level distribution of $\mathrm{Er}^{3+}$ is found in Figure I.

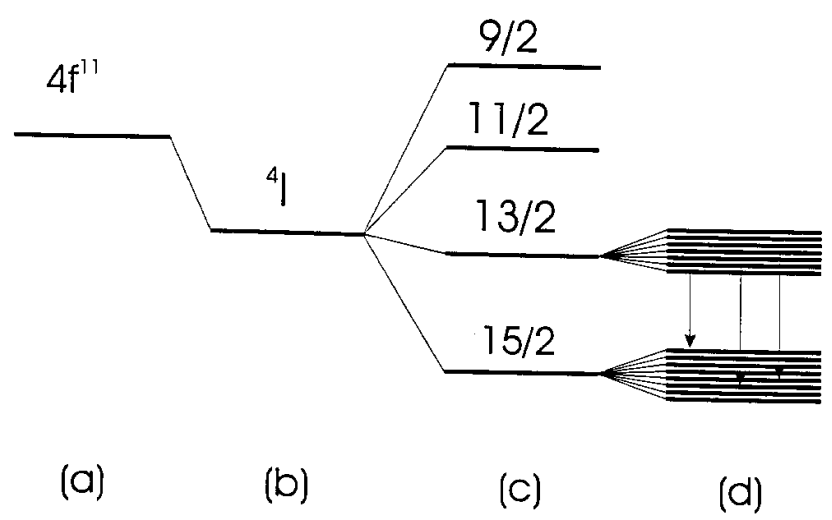

Figure 1. Schematic representation of (a) the $4 f^{11}$ states. (b) Electron-electron interaction. (c) Spin-orbit interaction. (d) Stark splitting. Some possible luminescent transitions are indicated.

In this paper I review the current status of Er doping of amorphous silicon, with emphasis in co-deposition techniques. Although the main subject is infra-red photoluminescence, other electronic as well as relevant structural properties are also covered.

\section{Preparation of Er doped a- Si:H}

\section{A. Ion Implantation}

Ion implantation has been used extensively as a means to incorporate $\mathrm{Er}$ in solids. Excellent review papers concerning erbium ion implantation for photonics[8] and about erbium ion implantation in silicon[9] are available. Er implantation in unhydrogenated a-Si[10] provided weak photoluminescence, detectable only at low temperatures. Er implantation on PECVD[11] or sputtered[12] samples, however, provided samples with room temperature photoluminescence after annealing at $300-850{ }^{\circ} \mathrm{C}$. The luminescence intensity was increased a factor 2 by oxygen coimplantation. Ion implantation in amorphous SIPOS (Semi-Insulating Poly Silicon, a silicon alloy containing up to 30 at.\% $\mathrm{O}$ and $\mathrm{H}$ ) yielded samples with higher luminescence intensity and lower temperature quenching than crystalline silicon[13]. Ion implantation is a well established technique, which provides good control of the concentration profile in the sample, an yielded the best electroluminescent devices to date in crystalline silicon[14]. However, because of the unavoidable damage associated with the implantation process, a high temperature annealing step is imperative. It is well known that annealing a-Si:H above $\sim 400{ }^{\circ} \mathrm{C}$ causes hydrogen effusion, which deteriorate the electronic properties of a-Si:H.

\section{B. Co-deposition}

In order to avoid the implantation damage due to ion implant, co-deposition techniques were developed by some research groups. These techniques can be roughly divided in variations around sputtering and PECVD, which are the most widely used processes for the preparation of electronic quality a-Si:H.

\section{Sputtering}

Sputtering is a deposition technique in which a-Si:H is formed from $\mathrm{Si}$ atoms removed from a $\mathrm{Si}$ target by momentum transfer from a cold plasma, normally of Ar. Molecular hydrogen is added to the sputtering gas to passivate the Si dangling bonds. The first report[15] of $\mathrm{Er}^{3+}$ luminescence from co-deposited a-Si:H〈Er $\rangle$ used rf-sputtering for sample preparation. Two targets, one of pure $\mathrm{Si}$ and one of pure Er were interchanged in the cathode during the deposition process, alternating 60 sec Si cycles with 5-10 sec Er cycles. The samples obtained presented a-Si:H photoluminescence at low temperatures and also $\mathrm{Er}^{3+}$ luminescence which was stronger than that from an $\mathrm{Er}^{3+}$ doped crystalline $\mathrm{Si}$ sample. Adding $\mathrm{Yb}^{3+}$ to a-Si:H using the same deposition technique did not result in $\mathrm{Yb}^{3+}$ luminescence.

Erbium photoluminescence (PL) in a-Si:H with much less temperature quenching was obtained in asdeposited samples by using the MASD[16], a modified sputtering technique. In this process[17], a metallic Er target is put in a dc-magnetron sputtering configuration, and the sputtering gas consists of an $\mathrm{Ar}+\mathrm{SiH}_{4}$ mixture.

More recently another possibility has been explored: rf-sputtering deposition from a Si substrate partially covered by small metallic Er platelets[18]. Using this procedure it is possible to control the Er concentration in the sample over a wide range. As-deposited samples present room-temperature $\mathrm{Er}^{3+} \mathrm{PL}$, although thermal annealing increases the PL efficiency. The deposition conditions that yield the strongest $\mathrm{Er}^{3+} \mathrm{PL}$ produce a-Si:H films with columnar morphology which readily react with ambient oxygen. This indicated that higher PL efficiency could be obtained if oxygen would 
be added to the material during deposition. This is easily done by adding a controlled leak to the deposition chamber $[19,20]$ and resulted in samples with optimized as-deposited room temperature PL.

\section{PECVD}

The best electronic quality a-Si:H films, adopted by the solar cell and TFT industry, are prepared using the PECVD technique. This has motivated studies of the preparation of a- $\mathrm{Si}: \mathrm{H}\langle\mathrm{Er}\rangle$ by this process. In this technique a-Si:H is formed from plasma decomposition of silane $\left(\mathrm{SiH}_{4}\right)$, in a much smoother process than sputtering. The resulting films have a density of states in the gap that can be one order of magnitude below that of comparable films obtained by sputtering. The main problem is the need of a suitable carrier to bring Er to the deposition chamber. The carrier gases are normally metalorganics, and the carbon contamination of the samples is unavoidable. Recently, the St. Petersburg group has been able to prepare Er doped a-Si:H by PECVD, using either $\operatorname{Er}(\mathrm{HFA})_{3} \cdot \mathrm{DME}[21]$ or $\operatorname{Er}(\mathrm{TMHD})_{3}[22]$. The latter found vibration modes in the infra-red absorption spectrum that were associated with carbon.

\section{Structural Properties}

\section{Composition}

Sample composition is often measured by Rutherford Backscattering Spectroscopy (RBS), which is a very appropriate technique for quantitative measurements of heavy impurities concentrations in light hosts. One important result, which is frequently disregarded is the presence of residual oxygen in a- $\mathrm{Si}: \mathrm{H}$, in concentrations in the 0.2 to 0.3 at. \% range $[16,11,19]$. This residual oxygen plays a very important role in determining the electronic properties of the material. Moreover, the presence of small oxygen partial pressures during co-sputtering inhibits the deposition rate of Er, most probably because of the high reactivity between these two elements.

\section{Local Properties}

Since the ${ }^{4} I_{13 / 2} \rightarrow{ }^{4} I_{15 / 2}$ transition probability is determined by the local environment of the $\mathrm{Er}^{3+}$ ions, the information about their chemical neighborhood is very important. Two local techniques have been used to study Er in a-Si:H: Mössbauer spectroscopy and EXAFS.

\section{Mössbauer Spectroscopy}

Mössbauer spectroscopy studies[23, 24] were performed in a-Si:H $\langle\mathrm{Er}\rangle$ samples prepared using the MASD technique[16]. The samples were irradiated with slow neutrons to become rich in ${ }^{169} \mathrm{Er}$ and ${ }^{169} \operatorname{Er}\left({ }^{169} \mathrm{Tm}\right)$ emission Mössbauer spectra were recorded. The results reveal the superposition of a singlet and a quadrupole doublet. The singlet is attributed to a silicon coordination shell and the quadrupole to a non-cubic oxygen neighborhood. In samples exhibiting $\mathrm{Er}^{3+} \mathrm{PL}$, the quadrupole signal is maximum, indicating that the optically active $\mathrm{Er}^{3+}$ is arranged within Er-O clusters.

\section{EXAFS}

EXAFS is a very useful technique to determine the coordination of Er in silicon. It was used to establish that in crystalline silicon luminescent $\mathrm{Er}^{3+}$ is coordinated with oxygen as in $\mathrm{Er}_{2} \mathrm{O}_{3}[25]$. In a-Si:H the situation is similar but the Er neighborhood has important differences[26]. It was shown[27] that in co-sputtered samples the first neighbor shell consists of oxygen atoms with an average coordination between 2 and 3.6, compared to 6 in $\mathrm{Er}_{2} \mathrm{O}_{3}$. Furthermore, the average Er-O separation is much smaller (up to $0.2 \AA$ ) than in $\mathrm{Er}_{2} \mathrm{O}_{3}$ and is minimum for an average coordination of 3 . The results indicate that $\mathrm{ErO}_{3}$ clusters are the energetically favored configuration of $\mathrm{Er}$ in a-Si:H. Being a strong oxygen getter, Er tends to be bonded to all the available residual O during deposition, and is incorporated to the a-Si:H network in the form of $\mathrm{ErO}_{x}$ clusters.

\section{Electronic Properties}

\section{Photoluminescence}

\section{Infra Red}

Most of the studies of a-Si:H $\mathrm{Er}\rangle$ have as ultimate goal the optimization of the photoluminescence intensity at room temperature. The $\mathrm{Er}^{3+}$ photoluminescence spectrum in a-Si:H consists of a characteristic double peak structure (Fig.2), which at room temperature is very similar to $\mathrm{Er}^{3+}$ in crystalline silicon. As the temperature is reduced the higher wavelength peak decreases in relation to the higher intensity, lower 
wavelength one. No additional structure can be resolved down to $2 \mathrm{~K}$ [28]. The spectral width of the main peak and the wide tails are due to Stark splitting of the excited and ground states, by the local electric field, which can be broadened homogeneously or inhomogeneously[7]. The exact position of the peaks depend on the oxygen content of the sample[11] and very weakly on the temperature [19]. The PL intensity depends on the oxygen content, and an optimum is found at around 10 oxygen atoms per erbium[19, 20]. This is probably related to the formation of $\mathrm{ErO}_{3}$ clusters[27], the remainder oxygen being bonded to silicon. The temperature dependence of the luminescence intensity is represented in Figure IV. The temperature quenching optimized samples between 20 and 300K[19], slightly greater than a factor 3 , is much smaller than in crystalline or in ion implanted amorphous silicon[8].

This quenching is much stronger above $70 \mathrm{~K}$.

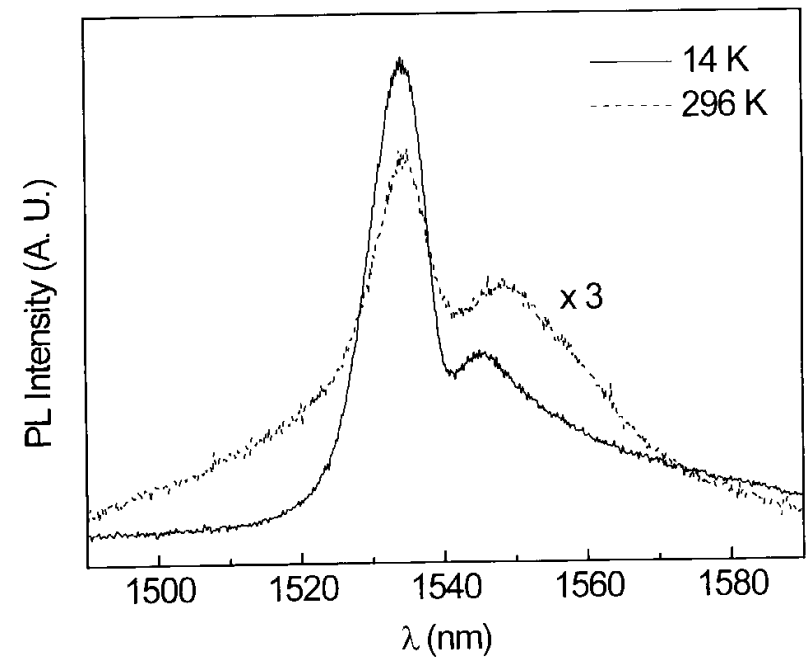

Figure 2. Typical $\mathrm{Er}^{3+}$ in a-Si:H photoluminescence spectra. The temperatures are indicated.

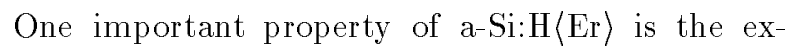
istence of an efficient process of excitation transfer from the host to the ions. Photoluminescence excitation curves[29] show that the PL intensity closely follows the absorption coefficient of a-Si:H. This is very important for electroluminescent devices applications. In glasses[30], the resonant excitation process is much more efficient than excitation transfer.

The whole photoluminescence process is then (i) absorption of a photon by the host, generating an electron-hole pair, (ii) transfer of the excitation to an $\mathrm{Er}^{3+}$ ion, (iii) radiative decay of the ion.

The $4 f$ states are $10-20 \mathrm{eV}$ below the valence states of a-Si:H. Two processes have been proposed for the ex- citation of rare earth impurities in semiconductors[31]: impact and Auger excitation, the probability of the latter increasing in the presence of localized states. Indeed, a Defect Related Auger Excitation (DRAE) mechanism[29, 32] in which Er ions are Auger excited with the capture of band-tail electrons by neutral defects is consistent with experimental results, in particular with the temperature quenching curves.

During any of the steps (i), (ii) or (iii) non-radiative loss of the excitation may occur. The recombination of an electron-hole pair in a defect not resulting in Auger excitation may take place during step (i). This is the reason why the PL intensity decreases if the samples are annealed at temperatures above $400^{\circ} \mathrm{C}$. Above this temperature, the intensity of infrared absorption by the Si- $H$ vibration mode at $\sim 650 \mathrm{~cm}^{-1}$ decreases [28, 33], indicating out-diffusion of hydrogen, which increases the density of dangling bonds.

There are controversial opinions concerning the main cause of temperature quenching of the PL. In implanted samples the quenching has been explained supposing an increase of the backtransfer rate of the excitation[11] (step iii), while in MASD samples it has been attributed to the decrease of the excitation rate [29] (step ii). Our own data[19] of PL intensity dependence on the laser intensity over 2 decades for cosputtered samples seems to support [29]. More studies are necessary to clear this point. It is our belief that the quenching mechanism should not depend on the form of sample preparation.

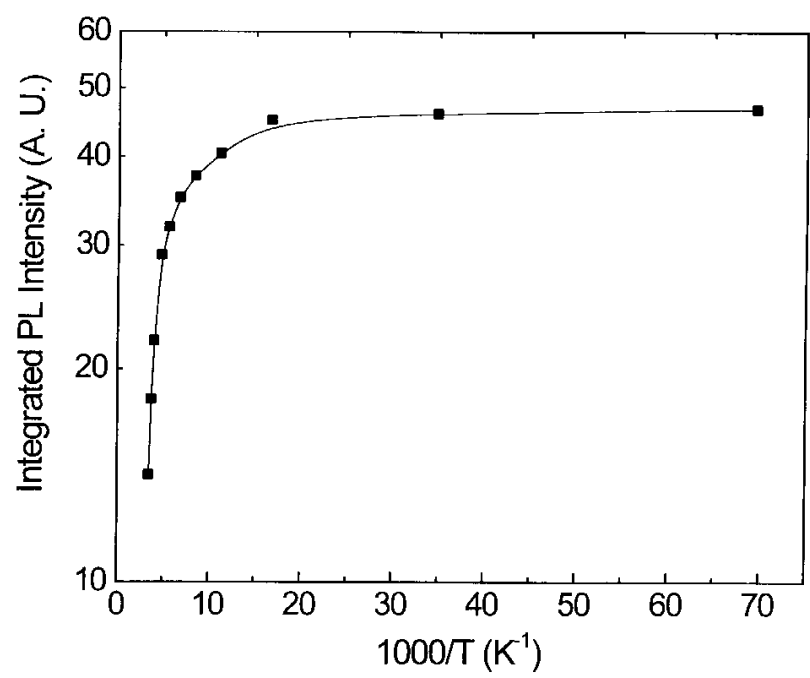

Figure 3. Temperature quenching of the $\mathrm{Er}^{3+}$ luminescence intensity. 


\section{Visible}

If the gap of the host is high enough, it is possible to observe transitions between higher excited states of $\mathrm{Er}^{3+}[34,35]$. This was achieved by adding as much as 10 at. \% Er to unhydrogenated a-SiN, with a high gap. Green luminescence $(\sim 545 \mathrm{~nm})$ was detected at room temperature corresponding to the ${ }^{4} S_{3 / 2} \rightarrow{ }^{4} I_{15 / 2}$ transition. Notice, however, that in this material the PL efficiency is probably small and unlike the case of a-Si:H〈Er $\rangle$, strong resonance excitation effects are observed, indicating that excitation transfer from the host is rather inefficient. Nevertheless, these results widen the range of potential applications of rare earth doped amorphous silicon based alloys.

\section{Electroluminescence}

$\mathrm{Er}^{3+}$ electroluminescence (EL) has been achieved in a-Si:H $\langle\mathrm{Er}\rangle$. A simple heterojunction diode configuration $n$-type $\mathrm{Cz}-\mathrm{Si} / \mathrm{a}-\mathrm{Si}: \mathrm{H}\langle\mathrm{Er}\rangle / \mathrm{Al}$ emits EL at $1.54 \mu \mathrm{m}$ when reverse biased in the breakdown region[32]. Crystalline silicon is transparent at this wavelength and EL can be detected from the substrate side. Under direct bias holes are injected in the a-Si:H $\langle\mathrm{Er}\rangle$ layer, transverse it and recombine radiatively at the crystalline substrate emitting near-band gap $1.2 \mu \mathrm{m}$ luminescence. No $\mathrm{Er}^{3+}$ luminescence is observed under direct bias. This result was reproduced in our laboratory, albeit our devices presented lower breakdown voltages[19]. No EL signal was detected at reverse bias voltages below the onset onset of breakdown, indicating that impact processes may be at least partially responsible for the excitation. The electric field and temperature dependence can be explained by the DRAE model[36]. The performance of these devices is quite poor, and more research is necessary before efficient devices can be developed.

\section{Doping Effects}

Doping of amorphous semiconductors is generally achieved by adding impurities of groups III or V of the periodic table. However, the addition of rare earth ions may have doping effects in a-Si:H in the sense of controlling the electronic properties of the material and determining the Fermi level position. From electrical equilibrium considerations, it would be expected that under a charge transfer doping mechanism $\mathrm{Er}^{3+}$ would be a donor in silicon. Early reports on crystalline silicon indicated $p$-type doping[37], while more recent results have found the expected $n$-type doping[38, 39]. In unhydrogenated a-Si, several different rare earths turned out to be acceptors and $p$-type doping was found[40]. In a recent work, $\mathrm{Er}^{3+}$ doping effects were measured in a-Si:H and this doping was classified as $n$-type, although no measurements were made to determine the majority carriers[29]. Thermopower measurements at room temperature[27] indicated $p$-type character. The question raised is why $\mathrm{Er}^{3+}$ is sometimes a donor and sometimes an acceptor. In fact this behavior results from the peculiar chemistry of $\operatorname{Er}[27]$. Being a strong oxygen getter, Er is incorporated to the network in the form of $\left[\mathrm{ErO}_{\delta}\right]^{+3-2 \delta}$ complexes, the most stable configuration being for $\delta=3$, as represented in Figure IV. For an average $\delta<1.5$ the complexes have donor behavior, whilst for an average $\delta>1.5$ they are acceptors. In a-Si:H, the usual Er concentrations and residual $\mathrm{O}$ content are such that $\delta>2$ and $p$-type doping results. Systematic doping studies with controlled oxygen concentration are needed to test this model. Charge transfer doping of amorphous semiconductors is a new subject of study.

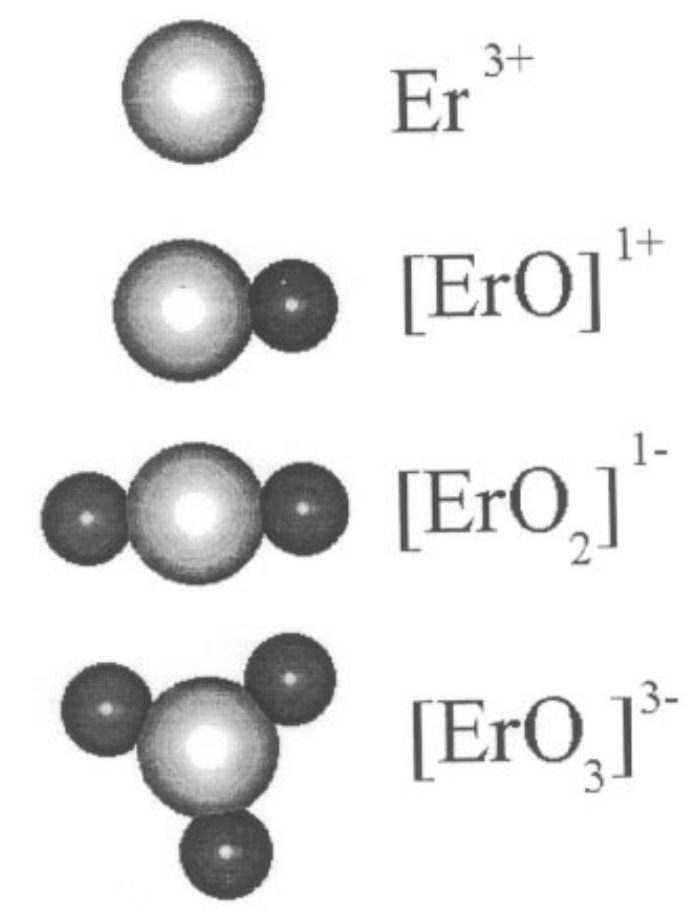

Figure 4. Schematic model of Er-O clusters that are formed in a-Si:H.

\section{Conclusions}

The study of a- $\mathrm{Si}: \mathrm{H}\langle\mathrm{Er}\rangle$ is rapidly becoming an important sub-area in amorphous semiconductors research. 
In this paper I reviewed relevant issues like preparation methods, structural and electronic properties, with emphasis in photoluminescence. Although the community has reached some degree of understanding, some fundamental topics remain open: What process determines the temperature quenching? What is the doping mechanism? Can higher photoluminescence and electroluminescence efficiencies be achieved? What is the exact role of oxygen? What can be expected from different amorphous semiconductor hosts? And from different rare earth dopants? And from different co-dopants?

There are apparently more open questions than answers. That is why I believe this is and will be a very exciting field of research in the years to come.

\section{Acknowledgements}

It is a pleasure to acknowledge all collaborators who actively participated in the activities concerning the aSi:H〈Er project: A. C. Iñiguez, C. Piamonteze and E. Horta, at UNICAMP, M. C. Martins Alves and H. Tolentino at LNLS, and A. R. Zanatta now at USPSão Carlos. M. Tabacniks from USP-São Paulo always helped us with the RBS measurements. Continuing support from my colleagues at the Laboratory of Photovoltaic Research and at GFURCO, UNICAMP is gratefully acknowledged. The discussions with I. Solomon at the very early stages of the project were very illuminating. This work would not have been possible without the financial support of CNPq, FAPESP and PRONEX "Fotônica em Telecomunicações".

\section{References}

[1] G. Styx, Scientific American, December 1998, pp. 9-10.

[2] G. P. Agrawal, Fiber-Optic Communication Systems, $2^{\text {nd }}$ edition, (John Willey, New York, 1997) p. 287.

[3] Rare Earth Doped Semiconductors, edited by G. S. Pomrenke, P. B. Klein and D. W. Langer, (MRS Symp. Proc. 301, Pittsburgh, 1993).

[4] Rare Earth Doped Semiconductors II, edited by A. Polman, S. Coffa, and R. Schwartz, (MRS Symp. Proc. 422, Pittsburgh, 1996).

[5] Silicon-base optoelectronics, MRS Bulletin 23 (4), April 1998.

[6] S. Hüfner, Optical Spectra of Transparent Rare Earth Compounds, (Academic Press, New York, 1978).

[7] E. Desurvire, Erbium Doped Fiber Amplifiers, (John Wiley, New York, 1994).

[8] A. Polman, J. Appl. Phys. 82, 1 (1997).
[9] S. Coffa, S. Lombardo, F. Priolo, G. Franzó, S. U. Campisano, A. Polman and G. N. van den Hoven, Il Nuovo Cimento 18D, 1131 (1996).

[10] J. S. Custer, E. Snoeks and A. Polman, in Phase Formation and Modification by Beam-Solid Interactions, edited by G. S. Was, L. E. Rehn and D. Follstaed, (MRS Symp. Proc 235, Pittsburgh, 1992) p. 51.

[11] J. H. Shin, R. Serna, G. N. van den Hoven, A. Polman, W. G. J. H. M. van Sark and A. M. Vredenberg, Appl. Phys. Lett. 68, 997 (1996).

[12] M. Kechouane, N. Beldi, T. Mohammed-Brahim, H. L'Haridon, M. Salvi, M. Gauneau and P. N. Favennec, in Rare Earth Doped Semiconductors, (ref. [3]), p. 133.

[13] G. N. van den Hoven, J. H. Shin, A. Polman, S. Lombardo and S. U. Campisano, J. Appl. Phys. 78, 2642 (1995).

[14] G. Franzò, S. Coffa, F. Priolo and C. Spinella, J. Appl. Phys. 81, 2784 (1997).

[15] T. Oestereich, C. Swialtowski and I. Broser, Appl. Phys. Lett. 56, 446 (1990).

[16] M. S. Bresler, O. B. Gusev, V. Kh. Kudoyarova, A. N. Kuznetsov, P. E. Pak, E. I. Terukov, I. N. Yassievich, B. P. Zakharchenya, W. Fuhs and A. Sturm, Appl. Phys. Lett. 67, 3599 (1995).

[17] V. Marakhonov, N. Rogachev, J. Ishkalov, J. Marakhonov, E. Terukov and V. Chelnokov, J. NonCryst. Sol. 137\&138, 817 (1991).

[18] A. R. Zanatta, L. A. O. Nunes and L. R. Tessler, Appl. Phys. Lett. 70, 511 (1997).

[19] L. R. Tessler and A. C. Iñiguez, in Amorphous Silicon Technology -1998, (MRS Symp. Proc. 507, Pittsburgh, PA 1998), in press.

[20] V. Kh. Kudoyarova, A. N. Kuznetsov, E. I. Terukov, O. B. Gusev, Yu. A. Kudriavtsev, B. Ya. Ber, G. M. Gusinskii, W. Fuhs, G. Weiser and H. Kuehne, Semiconductors 32, 1234 (1998) [Fiz. Tekh. Poluprovodn. 32, 1384 (1998)].

[21] V. B. Voronkov, V. G. Golubev, A. V. Medvedev, A. B. Pevtsov, N. A. Feoktisov, N. I. Gorshkov and D. N. Suglobov, Phys. Sol Stat. 40, 1301 (1998) [Fiz. Tverd. Tela (St. Petersburg) 40, 1433 (1998)].

[22] E. I. Terukov, O. I. Kon'kov, V. Kh. Kudoyarova, O. B. Gusev and G. Weiser, Semiconductors 32, 884 (1998) [Fiz. Tekh. Poluprovodn. 32, 987 (1998)].

[23] V. F. Masterov, F. S. Nasredinov, P. P. Seregin, V. Kh. Kudoyarova, A. N. Kuznetsov and E. I. Terukov, Appl. Phys. Lett. 72, 728 (1998).

[24] V. F. Masterov, F. S. Nasredinov, P. P. Seregin, E. I. Terukov and M. M. Mezdrogina, Semiconductors 32, 636 (1998) [Fiz. Tekh. Poluprovodn. 32, 708 (1998)].

[25] D. L. Adler, D. C. Jacobson, D. J. Eaglesham, M. A. Marcus, J. L. Benton, J. M. Poate, and P. H. Citrin, Appl. Phys. Lett. 61, 2181 (1992).

[26] L. R. Tessler, C. Piamonteze, A. C. Iñiguez, M. C. Martins Alves and H. Tolentino, in Applications of Synchrotron Radiation Techniques to Materials Science IV, (MRS Symp. Proc. 524, Pittsburgh, 1998), p. 327. 
[27] C. Piamonteze, A. C. Iñiguez, L. R. Tessler, M. C. Martins Alves and H. Tolentino, Phys. Rev. Lett. 81, 4652 (1998).

[28] L. R. Tessler and A. R. Zanatta, J. Non-Cryst. Sol. 227-230, 399 (1998).

[29] W. Fuhs, I. Ulber, G. Weiser, M. S. Bresler, O. B. Gusev, A. N. Kuznetsov, V. Kh. Kudoyarova, E. I. Terukov and I. N. Yassievich, Phys. Rev. B 56, 9545 (1998)

[30] W. J. Miniscalco, J. Lightwave. Tech., 9, 234 (1991).

[31] I. N. Yassievich and L. C. Kimerlingh, Semicond. Sci. and Technol. 8, 718 (1993).

[32] O. B. Gusev, A. N. Kuznetsov, E. I. Terukov, M. S. Bresler, V. Kh. Kudoyarova, I. N. Yassievich, B. P. Zakharchenya and W. Fuhs, Appl. Phys. Lett. 70, 240 (1997).

[33] A. R. Zanatta and L. A. O. Nunes, Appl. Phys. Lett. 71,3679 (1997)
[34] A. R. Zanatta and L. A. O. Nunes, Appl. Phys. Lett. 72, 3127 (1998).

[35] A.R. Zanatta, M.J.V. Bell and L.A.O. Nunes, Phys. Rev. B (in press).

[36] O. Gusev, M. Bresler, A. Kuznetsov, V. Kudoyarova, P. Pak, E. Terukov, K. Tsendin, I. Yassievich, W. Fuhs and G. Weiser, J. Non-Cryst. Sol. 227-230, 1164 (1998).

[37] H. Ennen, G. Pomrenke, A. Axman, K. Eisele, W. Haidl and J. Schneider, Appl. Phys. Lett. 46, 381 (1985).

[38] J. L. Benton, J. Michel, L. C. Kimerling, D. C. Jacobson, Y. -H. Xie, D. J. Eaglesham, E. A. Fitzgerald and J. M. Poate, J. Appl. Phys. 70, 2667 (1991).

[39] S. Libertino, S. Coffa, G. Franzó and F. Priolo, J. Appl. Phys. 78, 3867 (1995).

[40] J. H. Castilho, I. Chambouleyron, F. C. Marques, C. Rettori and F. Alvarez, Phys. Rev. B 43, 8946 (1991). 